\title{
Lidil
}

Revue de linguistique et de didactique des langues

$43 \mid 2011$

Le rapport au savoir dans les discours professionnels

\section{Mise en perspective des savoirs professionnels dans des discours de retour d'expérience. Étude contrastée de deux enseignantes de français langue étrangère}

Analysis of Professional Knowledge through Discourse on Experiential LearningInterviews. A Study of contrast

Véronique Rivière et Lucile Cadet

\section{OpenEdition} Journals

Édition électronique

URL : http://journals.openedition.org/lidil/3105

DOI : 10.4000/lidil.3105

ISSN : 1960-6052

Éditeur

UGA Éditions/Université Grenoble Alpes

Édition imprimée

Date de publication : 30 mai 2011

Pagination : 41-55

ISBN : 978-2-84310-201-1

ISSN : $1146-6480$

Référence électronique

Véronique Rivière et Lucile Cadet, « Mise en perspective des savoirs professionnels dans des discours de retour d'expérience. Étude contrastée de deux enseignantes de français langue étrangère », Lidil [En ligne], 43 | 2011, mis en ligne le 30 novembre 2012, consulté le 19 avril 2019. URL : http:// journals.openedition.org/lidil/3105; DOI : 10.4000/lidil.3105 


\title{
Mise en perspective des savoirs professionnels dans des discours de retour d'expérience. Étude contrastée de deux enseignantes de français langue étrangère
}

\author{
Véronique Rivière * et Lucile Cadet**
}

RÉSUMÉ

La présente contribution s'appuie sur deux formes d'entretiens d'autoconfrontation menés auprès de deux enseignantes de français langue étrangère, l'une, chevronnée, l'autre, débutante. Elle met en parallèle deux discours professionnels de retour d'expérience. Elle tente de mettre au jour par contraste la manière dont chacune mobilise ses savoirs professionnels pour interpréter une action d'enseignement après-coup. Elle s'attache à définir en quoi consistent ces savoirs professionnels, la façon dont chacune se positionne vis-à-vis d'eux dans le discours et les ressources discursives qu'elles mobilisent. L'étude montre que l'enseignante chevronnée ancre ses savoirs professionnels dans la situation de classe et donne une impression de maitrise de son activité. L'enseignante débutante ancre ses savoirs professionnels en se référant à des savoirs théoriques et en opérant un travail discursif de conceptualisation de son action. Cela nous amène finalement à poser la question de la professionnalisation des enseignants chevronnés par rapport à celle des débutants.

\section{ABSTRACT}

The present study is based on two types of interviews which were conducted of two instructors of "French as a foreign language", including one instructor who was considered as experienced and the other considered as novice. The study introduces parallel professional discourses based on differing experiential settings; instructors were offered the opportunity to view their filmed classroom settings and provide situational feedback. The study aims to highlight the contrasting manners

* ICAR, Université Lyon 2.

** THEODILE, IUFM, Université d'Artois. 
in which the respective instructors leverage their professional knowledge to interpret their teaching and instructional methods after the fact. The study attempts to determine the basis of the professional knowledge, that is to say, the manner in which each instructor positions herself vis-à-vis the experiences, and the discursive resources that the instructor employs in explaining her instructional methods. This study shows that the experienced instructor bases her knowledge in the situational context and gives the impression of mastery. The novice instructor, on the other hand, anchors professional knowledge in theory and utilizes discursive forms when explaining her actions. This leads us to question the degree of professionalization of experienced instructors as compared to that of novice instructors.

La présente contribution s'inscrit dans la problématique de la professionnalisation et des pratiques professionnelles des enseignants de français langue étrangère (désormais FLE). L'étude s'appuie sur deux entretiens d'auto-confrontation menés avec deux enseignantes de FLE et provoqués à des fins de recherche. Elle s'intéresse aux spécificités des discours professionnels de retour d'expérience que tiennent les enseignants sur leur pratique. Plus précisément, nous souhaitons montrer, à travers deux études de cas différenciés, la manière de rendre compte de son expérience, la manière de «montrer » certains savoirs professionnels et de les mobiliser pour interpréter une action d'enseignement après-coup.

En partant à la fois de la différence d'expérience entre les deux enseignantes et de la différence entre les deux dispositifs de verbalisation, cette étude nous amènera à identifier des ressources discursives de verbalisation des savoirs professionnels et ce que ces ressources nous apprennent du positionnement des enseignantes vis-à-vis de ces savoirs.

La première partie sera consacrée à contextualiser notre recherche et à définir notre objet d'étude. Nous exposerons ensuite une étude de cas différenciée basée sur une activité identique du point de vue de la séquentialité et sur laquelle les deux enseignantes reviennent : la mise en activité des apprenants ${ }^{1}$.

1. Il s'agit du moment où les deux enseignantes mettent au travail les apprenants : distribution du/des supports pédagogiques, énonciation des consignes nécessaires à la réalisation de la tâche, démarrage de l'activité, seul ou en groupe. 


\section{Préalables et contextualisation de l'étude}

\section{Les discours de retour d'expérience}

Ces discours s'inscrivent dans le champ des discours d'analyses de pratiques professionnelles, qu'ils soient sollicités pour la recherche ou pour la formation. Ils sont généralement convoqués selon trois dispositifs : l'entretien d'explicitation, l'auto-confrontation, l'instruction au sosie ${ }^{2}$. Dans une perspective de recherche en sciences du langage, on s'intéressera à l'activité langagière dans l'analyse du travail. Dès lors, les verbalisations de l'activité peuvent être considérées pour elles-mêmes, comme des «forme[s] d'activité en soi, régie[s] par des enjeux et des engagements propres, qui doivent faire eux-mêmes l'objet d'interprétations, de négociations, de régulations entre les partenaires» (Filliettaz et Bronckart, 2005, p. 9). Dans le cadre de la formation, il s'agit de faire parler les acteurs au travail et sur leur travail, de favoriser l'adoption d'une posture réflexive (Schön, 1983) afin de former des «praticiens réflexifs» ou «réfléchis» (Altet, 1996, p. 30-31), de provoquer certaines prises de conscience (psychologie du travail) et/ou d'apporter des améliorations au travail (ergonomie). En ce qui nous concerne, nous nous situons plutôt du côté de la caractérisation de ces discours du point de vue des savoirs et/ou savoir-faire qui y «émergent», de la manière dont les acteurs se positionnent vis-à-vis d'eux et de la façon dont ils les contextualisent explicitement ou non dans un environnement scientifique, historique, institutionnel ou socioprofessionnel ${ }^{3}$. Cette contextualisation nous parait être un élément fort de la professionnalisation des enseignants, dans la mesure où elle fait apparaitre les références sur lesquelles les enseignants se basent pour expliciter leur pratique.

2. Voir Filliettaz et Bronckart (2005) ou Bronckart et Bulea (2009) pour une explicitation de ces dispositifs méthodologiques.

3. Ce positionnement implique que le discours soit un lieu d'émergence, de mise en confrontation ou de construction des savoirs professionnels. Barbier et Galatanu (2004, p. 41-42) vont jusqu'à poser une relation d'équivalence entre discours et savoirs puisqu'ils définissent ces derniers comme «des énoncés propositionnels associés de façon relativement stable à des représentations ou à des systèmes de représentations sur le monde et sa transformation, faisant l'objet d'une reconnaissance sociale et d'un contrôle se situant dans le registre de la validité (épistémique : vrai/faux; pragmatique : efficace/inefficace), et considérés comme susceptibles d'être investis dans des activités de pensée, de communication ou de transformation de l'environnement». 


\section{Corpus}

Le corpus sur lequel s'appuie notre étude est issu de deux recherches universitaires ${ }^{4}$ visant à étudier la compétence de planification de deux enseignantes de FLE à des moments différents de leur carrière. En effet, la première étude s'est intéressée à Catherine (désormais C.), professeure chevronnée de vingt ans d'expérience ${ }^{5}$. La seconde a porté sur Valérie (désormais $\mathrm{V}$.), enseignante moins expérimentée qui comptabilisait, au moment de l'enquête, quatre années d'enseignement ${ }^{6}$. Les deux recherches comprennent des enregistrements audiovisuels et des transcriptions de cours de FLE ainsi que des enregistrements audio et des transcriptions d'entretiens d'auto-confrontation.

\section{Le recueil des données}

Durant les entretiens, les enseignantes visionnent leurs séances d'enseignement. Les discours qu'elles construisent sur leur pratique s'appuient donc sur un support mnésique. Il ne s'agit pas d'entretiens dirigés. Elles sont invitées à sélectionner et à commenter des extraits qu'elles choisissent au fur et à mesure du visionnage. Dans la première étude, les interventions de l'observatrice/enquêtrice étaient limitées volontairement à une activité de relance du type «là, pouvez-vous me dire ce qui se passe?». Dans la seconde recherche, quatre grandes questions ont été posées en amont des entretiens par l'enquêtrice :

- Comment as-tu structuré ton action?

- Comment as-tu fait pour que ça marche?

- Y a-t-il eu des entraves à l'action? ou des imprévus?

- Quelles ont été les sources de satisfactions/d'insatisfaction?

4. Recherches effectuées dans le cadre du master de recherche en didactique des langues, université Paris 3 : Hélène Ginabat, La planification dans l'agir enseignant et Laurence Corny, dossier réalisé dans le cadre du séminaire dirigé par Francine Cicurel : Agir professoral et pratiques de transmission, 2008-2009.

5. Il s'agit de cours de FLE dispensés, dans le cadre universitaire, à des étudiants chinois bénéficiant d'une année de mise à niveau en français avant d'intégrer un master de gestion et finance - cursus sciences économiques.

6. Les cours enregistrés ici sont des cours de FOS organisés à la demande d'une société de supermarchés que Valérie dispense bénévolement à des apprenants asiatiques salariés. 
Lors de la première recherche, les entretiens ont eu lieu «à froid», un mois après les enregistrements des cours. L'entretien qui s'est tenu dans le cadre de la seconde recherche a eu lieu «à chaud», deux heures environ après la fin du cours.

\section{Analyse des effets différentiels des protocoles d'enquête et de la situation d'interlocution}

Si les protocoles adoptés possèdent de nombreux points communs, ils révèlent aussi de grands décalages dans les conditions de recueil des données et dans le déroulement des entretiens. Ceci n'est pas sans incidence sur les données elles-mêmes et donc sur l'analyse que nous pourrons porter sur elles : la situation d'interlocution dans laquelle se trouve impliquée chacune des participantes exerce une influence sur le choix des actions commentées, sur le type de discours construit, sur l'identité socio-discursive montrée et élaborée par rapport aux attentes supposées des enquêtrices et par rapport à l'expertise que les enseignantes imaginent que les enquêtrices ont développée sur le métier d'enseignant. La relation entre les interlocutrices constitue sur ce point un facteur important d'influence. De nombreuses traces discursives de la présence de l'autre attestent de la relation que chacune des enseignantes construit avec son interlocutrice. V. et l'enquêtrice sont de statut égal et se connaissent bien (tutoiement, demandes de validation de ses propos ( «c'est ça hein»), marques de connivence sur des savoirs didactiques partagés $\left.{ }^{7}\right)$. Au contraire, C. et l'enquêtrice sont dans une relation inégalitaire du point de vue de l'âge, des fonctions et des expériences professionnelles (vouvoiement, peu de marques explicites de présence de l'enquêtrice dans le discours ${ }^{8}$ ) et l'organisation générale du discours de C. marque nettement cette inégalité. Ainsi, puisqu'elle s'est présentée initialement comme une enseignante chevronnée, C. a une image d'elle-même à défendre, du moins à ne pas dévaloriser. Pour

7. Comme dans l'expression utilisée par V. : «le fameux sens de lecture à dégager» qui indique que, dans leur échange, l'enseignante et l'enquêtrice s'appuient sur des références communes issues des enseignements auxquels elles ont été conjointement exposées à l'université.

8. Les extraits sélectionnés du discours de V. et C., de longueurs à peu près équivalentes, comptent huit énoncés à valeur phatique («tu vois», «tu remarqueras », «si tu veux») dans le discours de V. et trois seulement dans le discours de C. 
cela, elle se positionne comme quelqu'un qui sait analyser et parler de son action en alternant très régulièrement dans son discours mode évaluatif et mode argumentatif ${ }^{9}$.

\section{Études de cas}

\section{Modalités d'analyse}

Afin de réduire le rapport inégal et distant entre les deux cas étudiés, nous avons sélectionné des verbalisations au sujet d'un même moment de classe, l'entrée en activité, que les deux enseignantes ont choisi de commenter. Ce choix peut s'expliquer par l'enjeu important que recouvre cette étape pour l'ensemble de la séquence menée en classe et par son caractère particulièrement signifiant du point de vue de la compétence professionnelle de planification. Nous avons souligné ailleurs les enjeux de ce moment (engagement mutuel des acteurs, reconnaissance du contrat didactique) et les actions professorales auxquelles il donne lieu et les compétences spécialisées (communicationnelles et organisationnelles en particulier) nécessaires pour mener à bien cette phase du cours (Rivière, 2005 et 2006).

Dans les extraits retenus (interaction de classe et entretien), V. met en place puis commente une activité d'entrée dans la lecture d'un texte sur l'homéopathie ${ }^{10} ;$ C. introduit un exercice de classement lexical (classer des opinions de la plus positive à la plus négative).

À l'aide des outils de l'analyse des discours (prise en charge énonciative, types de discours et segments de discours, relations prédicatives, modalités), nous tentons d'identifier les traces linguistiques du rapport aux savoirs des deux enseignantes.

\section{Catherine : une mise en discours de la maitrise de son activité et de la situation}

\section{Une action valide et motivée}

La mise en activité des apprenants est l'occasion pour C. de souligner ses choix et les motifs de ses choix, les principes méthodologiques sous-tendant son action. Ainsi, entamer l'activité de tri lexical

9. Voir Rivière (2011) pour l'étude complète de ce phénomène.

10. Issu d'un guide de santé et se présentant sous forme de liste. 
(opinions positives-négatives) ne se fait pas au hasard, mais s'inscrit dans une progression didactique :

on va changer de sujet +++ (elle distribue des feuilles) on va dire son opinion on va continuer notre travail vous savez sur les stéréotypes etc. hein $\uparrow$ et les adjectifs [...] c'est la suite de hier (discours de classe) ${ }^{11}$.

Sur le plan discursif, cette validation de son action se lit, dans le commentaire, à travers l'axiologisation de son discours. C. met en relief et situe son action sur une échelle de valeurs («alors ce qui est important pour moi c'est de relier cet exercice à l'ensemble»). Mais son action devient également valide dès lors qu'elle en explicite les motifs tantôt tournés vers le passé («parce qu'ils doivent savoir qu’on lit un sujet»), tantôt tournés vers le futur ${ }^{12}$ («je relie cet exercice à l'ensemble pour qu'ils puissent classer leur feuille», «ça nous permet de d'augmenter notre vocabulaire»). L'activité didactique proposée répond donc à un ensemble de critères signifiants pour l'enseignante : besoins des apprenants et progression d'apprentissage. Par ailleurs, les nombreuses généralisations qui parsèment son discours fonctionnent comme des axiomes éprouvés contribuant à définir son métier («j’attends de voir s'ils sont bien embranchés dans l'exercice avant de me retirer un peu parce que bon on peut pas tenir pendant 3 heures sur la sellette + donc euh là je leur laisse la main »). En outre, C. montre la maitrise de son activité dans le passage discursif de l'action-occurrence à l'action typique, stable et prévisible. Par exemple, au tout début de sa prise de parole :

donc ce type d'exercice-là où on explique pourquoi on fait quelque chose c'est aussi non seulement un exercice de grammaire + mais on explicite on a un discours explicatif aussi c'est un exercice oral euh: qui qui n'a pas pour spécialement but de grammaire aussi $+j e$ mets souvent deux dimensions moi à mes exercices (début du film) (commentaire).

11. Conventions de transcription $:+$ et $++=$ pause. MAJUSCULE $=$ mot ou syllabe accentué. $::=$ allongement syllabique. $($ rire $)=$ commentaire du transcripteur sur l'activité non verbale. $\uparrow=$ Intonation montante. XXX = segment inaudible. Les éléments que l'on veut mettre en valeur sont en caractères italiques.

12. Nous reprenons la distinction désormais classique de Schütz (1987), en phénoménologie, au sujet de la structure temporelle de l'action humaine, entre les motifs d'action tournés vers l'expérience passée (motifs parce que) et ceux tournés vers la projection (motifs en vue de). 
Elle alterne d'abord «on» et «c'est + groupe nominal», puis vient le «je». Dans ce passage, c'est d'abord le métier qui parle (principe de la méthodologie d'enseignement constituée : définir les objectifs, le résultat attendu de l'activité). Puis C. semble se démarquer des pratiques communes («je... moi... mes») et par là même affirmer une identité plus personnelle de praticienne. Enfin la forte présence, dans l'ensemble de ses verbalisations, d'adverbes comme «souvent», «comme d'habitude», «toujours» renforce la typicalité et la prévisibilité de son action de mise au travail des apprenants.

\section{Mettre au travail les apprenants : contrôler la situation et agir en connaissance de cause}

Cette mise au travail et la textualisation que C. en propose donne l'occasion de scruter la mise en discours de sa connaissance aigüe des situations et des évènements. Ainsi, cette action d'enseignement spécifique semble passer par un contrôle intense de ce qui se passe pour chaque apprenant ou pour le groupe, mais aussi par une anticipation de micro-évènements qui surgissent. Le discours de C. est traversé par un certain nombre de verbes associés au sémantisme du regard, de la vérification («je m'assure avant», «j'attends de voir si», «voir si les étudiants ont capté»). De même, sur le mode de la traduction («je reste debout ça veut dire», «je leur donne une feuille pour deux, ça veut dire je la récupère»), elle interprète pour l'enquêtrice ou, plus globalement la communauté, son comportement et ses réactions face à ce qui se passe.

Enfin, dans ce commentaire,

XXX là je vous voyez voir si les étudiants ont capté ou alors ou bien ils posent une question + qu'est-ce que ça veut dire fabuleux + ou bien ils vont voir XXX parce que là c'est le premier exemple tout le monde n'est pas encore bien prêt euh j'essaye d'accélérer le mouvement tout en leur laissant XXX (commentaire),

C. donne à voir la manière dont se fabrique l'action professorale à grain fin (Cicurel, 2011). Celle-ci est en fait le résultat d'une prise d'indices, en rapport avec le comportement observable des élèves, énoncée ici sur le mode de l'alternative («ou bien ils posent une question... ou bien ils vont voir»), et sur le mode de l'évaluation de la situation à l'instant T («là c'est le premier exemple tout le monde n'est pas encore bien prêt»). Cette prise d'indices aboutit à une «réponse» professorale («j'essaie d'accélérer le mouvement tout en leur laissant...»). Finalement, C. se positionne comme une experte qui agit en connaissance de 
cause et de manière autonome, qui détient des savoirs sur les apprenants et sur les situations. Ses savoirs acquis au cours de l'expérience sont d'ordre méthodologique, plutôt personnels, sélectionnés et engrangés dans son répertoire didactique ${ }^{13}$ parce qu'ils permettent de produire une réponse didactique et pédagogique efficace et stable dans le temps. Le discours de C. témoigne d'une certaine binarité (alternative, traduction) entre l'action et sa signification et la mise en perspective des savoirfaire professionnels verbalisés dépend principalement de la situation de classe commentée.

\section{Valérie : contextualisation et conceptualisation de la confrontation au réel}

\section{Un discours articulé entre intentions, savoirs et situation de classe}

On assiste avec V. à la construction d'un discours centré sur une activité d'entrée en lecture où certains savoirs et savoir-faire de l'enseignant sont repérables.

\section{Savoirs cités, savoirs utilisés}

Premièrement, cette activité a un but et des motifs explicités face à l'enquêtrice, mais aussi face aux apprenantes : V. souhaite développer des stratégies de lecture concernant l'architecture des textes transférables dans la vie quotidienne :

savoir distinguer au niveau euh de de de la mise en page où était le symptôme qu'elles devaient chercher où était le remède + et la double organisation sur un plan comme ça par ordre alphabétique c'était la première liste + et la deuxième partie c'était un tableau où les choses étaient regroupées plutôt par les parties du corps (commentaire).

Deuxièmement, l'activité suit un déroulement par étapes que V. a l'habitude de mettre en place («en général», «comme d'habitude»)

13. Né sous la plume de Cicurel (2002, p. 157) et développé par Bigot et al. (2004), le répertoire didactique de l'enseignant se constitue au croisement de sa formation académique et pédagogique, de ses rencontres, de ses modèles et de son expérience. Il se situe ainsi à mi-chemin entre modèles de référence et pratique de classe «en temps réel». Ces deux pôles interagissant constamment entre eux, le répertoire didactique de chaque enseignant possède à la fois des traits partagés par l'ensemble de la communauté et des caractéristiques spécifiques à chacun. 
et s'appuie sur un certain nombre d'actions verbalisées à travers des consignes comme «regarder», «voir», «repérer» :

en général quand je les confronte à un texte je veux vraiment + qu'elles apprennent à se d'abord à se poser sur ce qu'elles comprennent enfin le fameux sens de lecture à dégager d'abord un truc un peu général et ensuite après je rentre dans le détail je réponds à leurs questions (commentaire)

donc je veux que vous regardiez regardiez un peu c'que + ça vous raconte + hein et vous allez me dire après je j'vous poserai une question parce qu'il faut comprendre comment ce texte est organisé $\uparrow[\ldots]$ comme d'habitude on va s'appuyer d'abord sur c'que vous connaissez d'accord + vous allez repérer + les mots que vous connaissez déjà +++ euh: + exemple +++ (classe)

Ces actions font référence à une technique d'accès au sens en langue étrangère bien identifiée en didactique du FLE. Il s'agit de «l'approche globale des textes écrits» définie en 1976 par S. Moirand et D. Lehmann, pionnières de la didactisation des textes fonctionnels. Cette technique est basée sur un certain nombre d'hypothèses didactiques et psycholinguistiques ${ }^{14}$. L'entrée dans un texte et la compréhension en langue étrangère reposent sur des activités de perception de signes, de repérage des indices formels, iconiques et thématiques, et sur la mobilisation des connaissances et expériences antérieures de l'apprenant. Elles nécessitent par conséquent un guidage fort de la part de l'enseignant dans l'univers sémiotique que représente le texte grâce notamment à des «questions clés» (Moirand, 1979, p. 24).

V. réfère plus exactement à une partie de cette technique dans la mesure où elle retient avant tout la première phase de cette démarche, la perception globale du texte par balayage. En effet, certaines actions attendues sont énoncées sur le mode de la déambulation lorsqu'elle est en classe et sur le mode de l'égarement durant l'entretien :

vous voyez $\mathrm{j}$ 'me promène dedans et $\mathrm{j}$ 'me dis tiens $\uparrow$ je vois ginko $[\ldots]$ là j'me promène pour vous montrer un p'tit peu le fonctionnement (classe)

mais au début j'veux vraiment à la limite qu'elles se perdent un peu seules et qu'elles s'aperçoivent en fait qu'elles ont de quoi se rattraper euh sans problème quoi [...] tellement peur qu'elles se noient (rires)

14. Pour plus d'informations concernant ces hypothèses, voir Moirand et Lehmann (1976) ou Moirand (1979). 
que ou qu'elles aient peur [...] en tout cas je ne les laisse pas assez mariner entre guillemets dans une espèce de + de sans repère il faudrait l'exercice ce s'rait ça ce s'rait qu'elles acceptent de s'perdre un peu et de se et de trouver après d'accord (commentaire).

Cela nous incite à penser que cette technique d'enseignement et les savoirs et savoir-faire didactiques et pédagogiques qui s'y rattachent sont plus cités qu'utilisés. V. en connait les principes et la rhétorique, mais pas la technique, qui se rapproche davantage, selon les termes qu'elle emploie, de la «perte de repères » que d'un guidage ferme.

\section{Approche globale des textes et situation de classe}

Cette approche, selon les dires de V., ne réussit pas complètement auprès des apprenantes. Le «mot à mot» dans lequel seraient «engluées » les apprenantes (logique du décodage-déchiffrage) du fait de leur culture éducative («mais c'est toujours tu vois ce réflexe de venir au MOT + qu'elles veulent absolument comprendre comme ça euh mot à mot quoi et ça je pense que ça fait partie d'la culture asiatique») vient contrecarrer son approche (relevé d'indices et déambulation dans le texte). Ce hiatus que l'on peut expliquer du fait de cette connaissance partielle de la technique et du niveau scolaire des apprenantes est très bien marqué sur le plan discursif lorsque V. commence l'entretien. Ainsi, dans cette portion où elle rend compte de ce qui se passe lorsqu'il y a «confrontation au texte» :

quand elles sont confrontées à un texte + il y a toujours alors elles ont un réflexe c'est qu'elles veulent tout comprendre chaque mot elles voudraient comprendre chaque mot et avancer dans leur lecture mot après mot [...] et deuxièmement donc en général quand je les confronte à un texte je veux vraiment + qu'elles apprennent à se d'abord à se poser sur ce qu'elles comprennent enfin le fameux sens de lecture à dégager (commentaire).

V. note la différence d'intentions entre les apprenantes et elle («elles veulent tout comprendre/je veux qu'elles apprennent à se poser sur que ce qu'elles comprennent»). En conséquence, dès le début de son entretien, V. construit un discours de déploration, empreint d'affects, plein de dévalorisation de soi et d'aveux d'incompétence («je n'attends pas assez c'est-à-dire que je devrais leur laisser plus de temps», «c'est mon défaut», «c'est quelque chose que je n'arrive pas à faire», «je le mène pas jusqu'au bout»). Pourtant, et même si l'on perçoit nettement un sentiment d'insécurité, à aucun moment V. ne remet directement en cause la démarche qu'elle applique. La situation d'interlocution dans 
laquelle elle se trouve, qui consiste en un recueil de discours sans aucun feedback de la part de l'enquêtrice - il n'y a aucune visée formative ${ }^{15}-$, ne lui permet pas d'aller jusque-là.

\section{Sous l'action, la conceptualisation?}

Il existe une certaine inadéquation entre les attentes de V. vis-à-vis du démarrage de l'activité et les conduites des apprenants. Face à cette situation, V. trouve cependant le moyen de faire progresser la séquence à travers ce qu'elle nomme «une forme d'exemplification» :

[...] personne ne se lance + et comme je m'identifie à elles j'me dis bon très souvent ça s'passe comme ça j'me jette à l'eau la première comme si (rires) j'étais + élève et même pas hein (rires) + donc je me mets un peu à un statut qu'est même pas je quitte le statut du prof + j'leur montre soi-disant l'exemple en disant bon allez + voilà moi j'me jette à l'eau alors voilà comment j'ferais + parce que là j'vais faire ça euh::: je cherche en fait je cherche pas hein + donc je j'vais mimer une scène où moi qui s'rais étrangère je chercherais quelque chose dans ma tête que je ne comprends pas voilà + et donc là je change mais ça c'est assez j'lai fait plusieurs j'l'ai fait souvent je + bon mais je pense que c'est une stratégie de de + c'est une sorte d'exemple + d'exemple $+j$ 'donne l'exemple + c'est une forme d'exempli+fication c'est ça? voilà ++ donc euh voilà c'que j'voulais dire (rires) + mais là j'fais un effort et le silence là du coup ça commence à m'angoisser (rires) (commentaire).

Le recours à la nominalisation est assez fréquent dans le discours de V. Ainsi, elle explique que face au silence («j'ai pas de réponses»), elle «quitte le statut de prof $[\ldots]$ et leur montre soi-disant l'exemple». Ce procédé est d'abord identifié et désigné par un présentatif («c'est une stratégie de de + c'est une sorte d'exemple + d'exemple:») puis est pris en charge par V. («+j'donne l'exemple $+»)$ et enfin est nominalisé («c'est c'est une forme d'exempli+fication c'est ça? voilà»). Cette nominalisation portant sur le processus agentif (Charaudeau, 1992) donne un aspect synthétique, abstrait et conceptuel à son action et, du même coup,

15. Rappelons que les discours d'auto-confrontation provoqués relèvent globalement des discours d'analyse de pratiques et visent à «ce que le formé se centre sur une tâche donnée, prenne conscience des diverses possibilités de réalisation de cette tâche ainsi que des diverses compétences qu'il mobilise à son égard, et qu'il enrichisse ce faisant les significations attribuées à son travail et à ses capacités » (Bronckart et Bulea, 2009, p. 51). 
attribue à celle-ci une certaine validité épistémique dont elle recherche la complétude auprès de sa camarade enquêtrice.

Nous n'avons pas rencontré chez $\mathrm{C}$. de tels procédés discursifs qui s'apparentent à une tentative de lier ce procédé pédagogique à un corps de savoirs savants.

\section{Discussion-conclusion}

Deux modes différenciés de relecture du vécu, qui est fonction de l'épaisseur de l'expérience et du mode de convocation des discours de retour d'expérience, apparaissent nettement dans la confrontation de ces deux cas :

C., qui a une grande expérience, construit un discours de maitrise de son action (motifs, échelle de valeurs de ses pratiques, généralisations) et des situations qu'elle vit (discours du contrôle). Elle parvient à expliciter la fabrication de son action, le mécanisme de prise de décision qui la sous-tend. $\mathrm{C}$. donne du sens à son action par la mise en relation des constituants de son action (les besoins des apprenants, ce qu'ils doivent savoir, les objectifs, l'adaptation au moment...). Cependant, elle ne contextualise pas sa pratique autrement que par le recours aux ressources propres à la situation qu'elle visionne. Elle pose sa pratique comme efficace et donne à voir, dans son discours, l'aisance de l'acteur.

V., moins expérimentée, contextualise ses savoir-faire plutôt explicitement dans le champ de la didactique du FLE même si elle n'a pas une connaissance approfondie de la technique d'enseignement à laquelle elle se réfère. On repère chez $\mathrm{V}$. un discours d'origine savante, issu des pratiques inculquées par l'université, totalement absent chez $\mathrm{C}$. V. trouve certaines réponses à ses insuffisances (se mettre à la place des apprenantes et faire un exemple) et le discours de retour d'expérience lui permet de conceptualiser cette réponse et de définir de manière personnelle des descripteurs de son action.

Cela nous conduit à interroger la professionnalisation au fil du temps. Nous avons deux enseignantes situées à un moment différent de leur carrière. La place des savoirs d'origine savante et d'origine expérientielle et situationnelle n'est pas la même dans les discours respectifs des deux enseignantes. Est-ce qu'une fois l'aisance professionnelle acquise, les savoirs savants qui assoient la pratique s'effacent derrière les savoirs d'expérience? Où se situe la fin de la professionnalisation des acteurs de l'enseignement? En d'autres termes, si la professionnalisation des débutants, la façon dont ils entrent dans le métier est 
très explorée, on peut se demander comment les enseignants dits chevronnés continuent d'évoluer.

\section{RÉFÉRENCES BIBLIOGRAPHIQUES}

Altet M. (1996) : «Les compétences de l'enseignant professionnel. Entre savoirs, schèmes d'action et adaptation : le savoir-analyser», dans L. Paquay, M. Altet, E. Charlier et P. Perrrenoud (dir.), Former des enseignants professionnels. Quelles stratégies? Quelles compétences?, Bruxelles, De Boeck, p. 27-40.

Barbier J.-M. et Galatanu O. (2004) : Les savoirs d'action. Une mise en mots des compétences, Paris, L'Harmattan.

Bigot V., Blondel E., Cadet L. et Causa M. (2004) : «La construction du répertoire d'enseignement lors du passage du statut d'étudiant à celui d'enseignant de FLE», Marges linguistiques, $n^{\circ}$ 7, Saint-Chamas, M.L.M.S Éditeur, consultable en ligne.

Bronckart J.-P. et Bulea E. (2009) : «Praticien réflexif ou praticien discursif?», Éducation Canada, no 49, p. 50-54.

Charaudeau P. (1992) : Grammaire du sens et de l'expression, Paris, Hachette.

Cicurel F. (2002) : «La classe de langue un lieu ordinaire, une interaction complexe», AILE, n ${ }^{\circ} 16$, Paris, p. 145-164.

— (2011) : «Le dire sur le faire : un retour (possible?) sur l'action d'enseignement», dans V. Bigot et L. Cadet (éd.), L'agir professoral : de l'interaction à la mise en discours de l'action, Paris, Riveneuve Éditions.

Filliettaz L. et Bronckart J.-P. (éd.) (2005) : L'analyse des actions et des discours en situation de travail, Louvain, Peeters.

Moirand S. et Lehmann D. (1976) : «L'approche globale des textes écrits», Études de Linguistique Appliquée, $\mathrm{n}^{\circ} 23$, Paris, Didier.

MoIrand S. (1979) : Situations d'écrit, Paris, CLE International.

RiviÈre V. (2005) : «Aujourd'hui nous allons travailler sur...» ou se mettre au travail en classe de langue : quelques aspects praxéologiques des interactions didactiques », Le français dans le monde, Recherches et Applications, Paris, CLE International, p. 96-104.

- (2006) : L'activité de prescription en contexte didactique. Analyse psycho-sociale, sémio-discursive et pragmatique des interactions en classe de langue étrangère et seconde, thèse pour le doctorat en didactique des langues et des cultures, université Paris 3-Sorbonne Nouvelle.

- (2011) : «Comment se saisir des discours sur l'agir enseignant? Quelques repères et outils conceptuels d'analyse de l'activité discursive », 
dans V. Bigot et L. Cadet (éd.), L'agir professoral : de l'interaction à la mise en discours de l'action, Paris, Riveneuve Éditions.

$\mathrm{S}_{\mathrm{CHÖN}}$ D. (1983) : The reflective practitioner, New York, Basic Books.

Schüтz A. (1987) : Le Chercheur et le Quotidien. Phénoménologie des sciences sociales, Paris, Méridiens Klincksieck. 